\title{
Determining the neutron star surface magnetic field strength of two $\mathrm{Z}$ sources
}

\author{
Guoqiang Ding ${ }^{1}$, Chunping Huang ${ }^{1,2}$, and Yanan Wang ${ }^{1,2}$ \\ ${ }^{1}$ Xinjiang Astronomical Observatory, Chinese Academy of Sciences, \\ 150, Science 1-Street, Urumqi, Xinjiang 830011, China \\ email: dinggq@gmail.com \\ ${ }^{2}$ University of Chinese Academy of Sciences, \\ China
}

\begin{abstract}
From the extreme position of disk motion, we infer the neutron star (NS) surface magnetic field strength $\left(B_{0}\right)$ of Z-source GX $17+2$ and Cyg X-2. The inferred $B_{0}$ of GX $17+2$ and Cyg X-2 are $\sim(1-5) \times 10^{8}$ G and $\sim(1-3) \times 10^{8}$ G, respectively, which are not inferior to that of millisecond X-ray pulsars or atoll sources. It is likely that the NS magnetic axis of Z sources is parallel to the axis of rotation, which could result in the lack of pulsations in these sources.
\end{abstract}

Keywords. Compact object, Neutron star, Accretion disk

\section{Introduction}

The neutron star (NS) surface magnetic field strength $\left(B_{0}\right)$ could be a critical parameter responsible for the behaviors of NS X-ray binaries (NSXB). However, it is difficult to measure $B_{0}$ directly. Nevertheless, it is feasible to estimate $B_{0}$ from some observable phenomena, such as quasi-periodic oscillations (QPOs) (e.g. Fock 1996) or "propeller" effects (e.g. Zhang et al. 1998), or inferred from theoretical models (Zhang \& Kojima 2006). From previous studies, it is believed that $B_{0}$ is larger in $\mathrm{Z}$ sources than in atoll sources (ref. Fock 1996, Zhang et al. 1998, Chen et al. 2006, Ding et al. 2011) or millisecond X-ray pulsars (ref. Cackett et al. 2009, Di Salvo \& Burderi 2003). Unlike in millisecond $\mathrm{X}$-ray pulsars or atoll sources, pulsations have never been observed in Z sources, why?

\section{Method and Data Analysis}

As discussed by Zhang et al. (1998), during the regular accretion state the inner disk radius $\left(R_{\mathrm{in}}\right)$ of an NSXB, which is equal to the magnetospheric radius $\left(R_{\mathrm{m}}\right)$ (Lamb et al. 1973), will vary between the radius of the innermost stable circular orbit (ISCO) and the corotation radius $\left(R_{\mathrm{c}}\right)$. Therefore, assuming $R_{\mathrm{in}}=R_{\mathrm{c}}$, we will get the upper limit of $B_{0}$, because more magnetic pressure is needed to push the accretion disk further, having

$$
B_{0, \max }=1.5 \times 10^{12}\left(\frac{\dot{M}_{\mathrm{m}}}{\dot{M}_{\mathrm{Edd}}}\right)^{1 / 2}\left(\frac{M_{\mathrm{ns}}}{1.4 M_{\odot}}\right)^{5 / 6}\left(\frac{R_{\mathrm{ns}}}{10^{6} \mathrm{~cm}}\right)^{-3} f^{-7 / 6} \xi_{1}^{-7 / 4} \mathrm{G},
$$

where $f$ is the NS spin frequency and $\xi_{1}$ is a coefficient in the range of $0.87-0.95$ (Wang 1995). Similarly, letting $R_{\text {in }}=R_{\mathrm{ISCO}}$, we will obtain the lower limit of $B_{0}$, because less magnetic pressure is needed to balance the gas pressure from the disk, getting

$$
B_{0, \min }=2.8 \times 10^{8}\left(\frac{\dot{M}_{\mathrm{m}}}{\dot{M}_{\mathrm{Edd}}}\right)^{1 / 2}\left(\frac{M_{\mathrm{ns}}}{1.4 M_{\odot}}\right)^{2}\left(\frac{R_{\mathrm{ns}}}{10^{6} \mathrm{~cm}}\right)^{-3} \xi_{1}^{-7 / 4} \mathrm{G} .
$$


With software HEASOFT 6.11 and FTOOLS V6.11, we use the RXTE observations made during Oct. $3-12$, 1999, a total of $\sim 297.6 \mathrm{ks}$ for GX $17+2$ and those during Jul. 2-7, 1998 , a total of $\sim 42 \mathrm{ks}$ for Cyg X-2 to perform our analysis. We divide the track on the hardness-intensity diagrams (HIDs) into some regions and then produce the spectrum of each region. XSPEC version 12.7 is used to fit the spectra with spectral models. From the spectral fitting parameters, we calculate mass accretion rates. Then, with the known NS mass and radius, making use of the above equations, we calculate the limits of $B_{0}$.

\section{Result and Discussion}

With mass accretion rates and $f_{\max }=584 \mathrm{~Hz}$ for GX $17+2$ and $f_{\max }=658 \mathrm{~Hz}$ for Cyg X-2 (Yin et al. 2007), we infer the limits of $B_{0}$ and get $\left(1 \leqslant B_{0} \leqslant 5\right) \times 10^{8} \mathrm{G}$ and $\left(1 \leqslant B_{0} \leqslant 3\right) \times 10^{8}$ G for GX $17+2$ and Cyg X-2, respectively, which are higher than the reported $B_{0} \sim(0.3-1) \times 10^{8} \mathrm{G}$ of atoll source Aql X-1 (Zhang et al. 1998), compatible with $B_{0} \sim(1-5) \times 10^{8} \mathrm{G}$ of accreting millisecond X-ray pulsar SAX J1808.4-3658 (Di Salvo \& Burderi 2003), but lower than $B_{0} \sim(1-3) \times 10^{9} \mathrm{G}$ of the first transient $\mathrm{Z}$ source XTE J1701-462 (Ding et al. 2011) or $B_{0} \sim(1-8) \times 10^{9}$ G of Cir X-1 (Ding et al. 2006).

Since the $B_{0}$ of $\mathrm{Z}$ sources is not inferior to that of millisecond X-ray pulsars or atoll source, pulsations should have been observed in the former, as in the latter. However, pulsations have not been detected in Z sources, why? Pringle \& Rees (1972) proposed that the NS pulsation emission would depend on the shape of emission cone, the orientations of the magnetic and rotation axes, and the line of the sight, and, furthermore, Lamb et al. (1973) suggested that the detected pulsation could be resulted from the condition that the NS magnetic axis does not coincide in direction with the axis of rotation. Therefore, it is likely that the NS magnetic axis of Z sources is parallel to the axis of rotation, or the orientation of the rotation axis is in agreement with the line of the sight, any of which could result in lack of pulsations in these sources.

\section{Acknowledgements}

This work is supported by the National Basic Research Program of China (973 Program 2009CB824800) and the Natural Science Foundation of China under grant no. 11143013.

\section{References}

Cackett, E. M., Altamirano, D., Patruno, A., Miller, J. M., Reynolds, M., Linares, M., \& Wijnands, R. 2009, ApJ, 649, L21

Chen, X., Zhang, S. N., \& Ding, G. Q. 2006, ApJ, 650, 299

Ding, G. Q., Zhang, S. N., Li, T. P., \& Qu, J. L. 2006, ApJ, 645, 576

Ding, G. Q., Zhang, S. N., Wang, N., Qu, J. L., \& Yan, S. P. 2011, AJ, 142, 34

Di Salvo, T. \& Burderi, L. 2003, A\& $A$, 397, 723

Fock, W. B. 1996, ApJ, 470, L127

Lamb, F. K., Pethick, C. J., \& Pines, D. 1973, ApJ, 184, 271

Pringle, J. E. \& Rees, M. J. 1972, A\& $A$, 21, 1

Wang, Y. M. 1995, ApJ, 449, L153

Yin, H. X., Zhang, C. M., Zhao, Y. H., Lei, Y. J., Qu, J. L., Song, L. M., \& Zhang, F. 2007, $A \mathscr{E} A, 471,381$

Zhang, C. M. \& Kojima 2006, MNRAS, 366, 137

Zhang, S. N., Yu, W., \& Zhang, W. 1998, ApJ, 494, L71 